\title{
La comunicación de los problemas de matemáticas en la didáctica de los Grados de Educación en la $\mathrm{UIC}^{*}$
}

\author{
Salvador VIDAL RAMÉNTOL \\ Universitat Internacional de Catalunya \\ svidal@uic.es \\ María Carmen BALAGUER FÀBREGAS \\ Universitat Internacional de Catalunya \\ mcbalaguer@uic.es
}

Recibido: $24 / 10 / 2012$

Aceptado: 23/01/2013

\section{Resumen}

El presente artículo presenta una propuesta experimental sobre cómo gestionar las demandas de los problemas matemáticos en la enseñanza primaria. En primer lugar, se describe el estado de la cuestión, poniendo énfasis en los aspectos atencionales y de motivación en el aula. En, segundo lugar, se presentan los resultados de una investigación en la que se implementó una metodología de enseñanza y aprendizaje de las matemáticas, fundamentado en un tratamiento estratégico de la lectura de las demandas de los problemas.

Palabras clave: Atención, motivación, demandas de problemas, lectura estratégica, contextualización.

\section{Communication Problems in Teaching of Mathematics Grade Education in the UIC}

\begin{abstract}
This paper presents an experimental proposal on how to manage the demands of mathematical problems in primary. First, it describes the state of the question, emphasizing aspects of attentional and motivation in the classroom. In second place, we present the results of a study in which we implemented a methodology of teaching and learning of mathematics, based on a strategic treatment for reading problems demands.

Keywords: Attention, motivation, problems demands, strategic reading, contextualization.

\section{Referencia normalizada}

VIDAL RAMÉNTOL, Salvador y BALAGUER FÀBREGAS, M Carmen (2013): "La comunicación de los problemas de matemáticas en la didáctica de los Grados de Educación en la UIC". Estudios sobre el Mensaje Periodístico. Vol. 19, Núm. especial marzo, págs.: 531-541. Madrid, Servicio de Publicaciones de la Universidad Complutense.
\end{abstract}

Sumario: 1. Introducción; 1.1. Estado de la cuestión; 1.2. Estrategias para llevar a cabo en el aula; 1.2.1. Estrategias de la conquista de la atención; 1.2.2. Estrategias de conquista de la participación; 1.3. Técnicas de motivación en el aula; 1.3.1. Recomendaciones. 2. Metodología. 3. Desarrollo del programa de Lectura estratégica de los problemas matemáticos (PLEPM). 4. Conclusiones. 5. Referencias bibliográficas

\section{Introducción}

Una de las dificultades que más tenemos que vencer en el aula de matemáticas es la resolución de problemas. Por eso, después de una investigación llevada a cabo a lo largo de un curso escolar queremos compartir las pautas que nos han llevado a un

* UIC: Universitat Internacional de Catalunya 
cambio cualitativo que favorezca la comprensión de los problemas y haga tener más conciencia del aprendizaje. Se trata de proponer un cambio actitud, con el objetivo de favorecer el aprendizaje y tomar conciencia del proceso individual de aprendizaje. Se trata de intentar dar a los maestros unos mecanismos que les permitan enseñar a sus alumnos a hacerse responsables de aquello que aprenden, de cómo lo aprenden i autoevaluarse del aprendizaje.

\subsection{Estado de la cuestión}

Hablamos de comprensión de los aprendizajes porque el objetivo es valorar los resultados de la implementación de un programa de lectura estratégica. La relación entre lectura y comprensión es muy estrecha, por tanto creemos que es muy interesante insistir en los textos para favorecer la lectura y la compresión de los problemas.

Con el Currículo actual redactado en base a que los alumnos desarrollen la competencia matemática es importante que cada niño o niña sepa discernir cuales son los conocimientos que necesita ante cualquier situación que se le presente. Para poder seleccionar la información necesaria hace falta una muy buena habilidad lectora. Veremos cómo hay una estrecha relación entre la comprensión lectora y la resolución de problemas matemáticos.

Para progresar en esta competencia proponemos una serie de estrategias de aprendizaje que ayuden al docente para que sus alumnos mejoren la atención y la participación como fuente de motivación y con ello incremento de la comprensión de los enunciados de los problemas. Es importante la actitud que el alumno adquiere respecto a la asignatura.

En el marco teórico son interesantes los principios de Keuworthy (en Gairín, 1990) sobre la formación y educación de las actitudes.

- Las actitudes se forman, refuerzan o cambian mejor cuando una persona tiene seguridad en sí misma y es capaz de aceptar los cambios.

- La mejor parte de las actitudes básicas se forman a los pocos años de vida.

- Las actitudes se pueden cambiar en cualquier edad.

- Las épocas de crisis personal y social producen cambios de actitud.

- Los cambios se producen mejor cuando todo el grupo está afectado. Esto produce seguridad a los individuos porque también cambian los demás.

- Las actitudes se cambian más fácilmente cuando los sujetos tienen la oportunidad de actuar según sus creencias.

- Pertenecer a nuevos grupos ayuda a reforzar los cambios de actitud.

- El testimonio de personas de categoría ayuda a las demás al cambio de actitudes.

- Las reuniones de mucha gente y otras situaciones llenas de emotividad ayudan a cambiar las actitudes, aunque su duración puede ser de poco tiempo.

- Si tocamos el orgullo o la necesidad práctica podemos ayudar en la formación o en el cambio de actitudes.

- La formación que llega de fuentes fiables, de toda confianza, especialmente si la descubre la persona que tiene que cambiar, puede facilitar los cambios.

- Los medios de comunicación de masas pueden tener una poderosa influencia en los cambios de actitud. 
En la didáctica de las matemáticas la actitud tiene un peso específico; J. Gairín (1990) afirma: "La actitud puede considerarse causa y efecto del aprendizaje. La actitud es una de las variables que intervienen en el aprendizaje de manera que actitudes negativas dificultan el aprendizaje, pero también podemos decir que una enseñanza mal administrada puede generar actitudes negativas en los alumnos".

El alumno debe ver que lo que se explica la puede ser útil, que es práctico o que se lo puede pasar bien; Mager (1981) se pregunta: "Si es valioso enseñar, ¿no lo es también esforzarse porque esa enseñanza se emplee?; la probabilidad de que un alumno ponga en práctica sus conocimientos es afectada por su actitud a favor o en contra de la asignatura; lo que disgusta tiende a olvidarse". Mager explica que hay cinco áreas de observación en relación a las actitudes y a su mejora: el ambiente escolar; el maestro; los materiales y los recursos didácticos; el ambiente físico, y las políticas administrativas.

La conducta del maestro incide en la del alumno. De este modo, cuanto más positivas son las percepciones de los alumnos hacia los sentimientos y expectativas de sus maestros, mejor es su rendimiento académico y más aceptable su conducta.

También debemos tener en cuenta los efectos que los ambientes escolares tienen sobre las actitudes y aprendizajes de los escolares. Se deben destacar los resultados obtenidos por Moos (1979).

a) Las clases orientadas a la relación y a la motivación pueden aumentar la satisfacción y el interés del estudiante por la materia, a la vez que mejoran su desarrollo personal (independencia, autoestima y creatividad).

b) Las clases orientadas al control llevan a la insatisfacción y no facilitan el desarrollo personal, social o académico.

Sobre el mismo tema (Wolf et al., 1981; Walberg et al., 1981; Baba y Fraser, 1983) varios autores señalan que las actitudes de los estudiantes hacia el ambiente de la clase están fuertemente relacionadas con el aprendizaje y también que el clima de clase crea una fuerte influencia sobre las actitudes individuales.

\subsection{Estrategias para llevar a cabo en el aula (Vidal, 2009)}

Existen un conjunto de posibles estrategias prácticas para llevar a cabo una motivación correcta. La motivación en clase abarca dos grandes niveles: el primero consiste en conquistar la atención del alumno; el segundo va más allá y requiere que los alumnos participen en el desarrollo de la clase.

\subsubsection{Estrategias de la conquista de la atención}

Comunicar la justificación racional de los objetivos consiste en aclarar a los alumnos la importancia y las razones de la consecución de un objetivo. Puede realizarse de dos modos distintos:

a) Indicando las consecuencias inmediatas o a largo plazo de la obtención del objetivo, de modo que el alumno vea su utilidad.

b) Haciendo preguntas a los alumnos, de modo que sean ellos mismos los que deduzcan la eficacia de la consecución de los objetivos: 
- Despertar la curiosidad

- Crear disonancia o choque

- Modificar el medio físico de aprendizaje

- Variar las pautas de instrucción

- Variar los canales sensoriales

- Usar el movimiento

- Usar sistemas de comunicación de forma matizada

\subsubsection{Estrategias de conquista de la participación}

- Interrogatorio: por definición, las preguntas buscan respuestas o, lo que es lo mismo, participación. Estas preguntas, siempre que sea posible, tienen que ser adecuadas a cada uno de los individuos; así daremos la oportunidad de que sean contestadas correctamente. También es necesario realizar preguntas que requieran la opinión de los alumnos.

- Refuerzo positivo:

- Refuerzo verbal simple: consiste en simples palabras que el maestro utiliza para elogiar la conducta del alumno.

- Refuerzo verbal complejo: el maestro utiliza una frase en la que, además de elogiar al alumno, indica el motivo del elogio.

- Refuerzo no verbal: en este caso el maestro se sirve del gesto para aprobar la respuesta emitida por el alumno. Puede acompañar los refuerzos verbales o ser utilizado aisladamente.

- Realimentación

- Creciente expectativa de éxito

- Representación de roles

\subsection{Técnicas de motivación en el aula}

- Correlación con la realidad: con ella el maestro procura establecer relación entre lo que enseña y la realidad circundante, con las experiencias de vida del alumno o con hechos de la actualidad. Esta técnica se confunde con la enseñanza concreta y tiene la virtud de dar sentido de realidad y autenticidad en las clases.

- Victoria inicial: se conduce al alumno a responder preguntas relativamente fáciles, pero comprometidas, presentadas con aspecto de difíciles. Naturalmente, el alumno responderá con exactitud y, entusiasmado con el éxito, sigue con más atención las tareas de la clase.

- Fracaso inicial: aquí hacemos preguntas aparentemente fáciles y eso provoca respuestas erróneas. Estas preguntas deben ir dirigidas preferentemente a los alumnos más capaces. Los otros despertarán ante el fracaso de los mejores y estos, a su vez, quedarán intrigados por sus errores inesperados.

- Problemática de las edades: el maestro debe relacionar el asunto a tratar con los problemas propios de cada fase de la vida (problemas de profesión, economía, cuestiones sociales y políticas, prestigio social...).

- Acontecimientos actuales de la vida social: el maestro parte de acontecimientos que pasan en la vida diaria. Es un enfoque de facilísima aplicación ya que, en cierto 
modo, todas las cuestiones se adhieren a las disciplinas enseñadas. Debe existir bastante preocupación docente para el uso de esta técnica porque es un medio excelente de dar sentido y funcionalidad a las clases, se vitalizarán y contribuirán al interés de la clase.

- Participación del alumno: el maestro, mediante interrogatorios y las situaciones problemáticas interesantes, hace que los alumnos tomen parte en las tareas escolares. Su preocupación debe ser la de sustraer al alumno de la situación de mero espectador para transformarlo en participante y realizador de la clase.

- Autosuperación: el maestro incentiva al alumno para que mejore su actuación haciéndole comparar la marcha de su aprendizaje en distintos momentos o con el rendimiento medio de la clase -pero no directamente con otros compañeros.

- Voluntad de aprobación: el maestro multiplica su atención en torno al comportamiento de sus alumnos utilizando la tendencia humana de preocuparse por la aceptación de lo que se está a punto de realizar, tanto por parte de los compañeros como del maestro. Así, el alumno se siente observado y aspira a mostrarse eficiente en la disciplina del maestro que lo ha notado con el objetivo de obtener, cada vez con un grado más alto, la aprobación y admiración del maestro.

- Elogios y censuras: los elogios y las censuras pueden funcionar como procesos motivadores si se usan inteligente y oportunamente. Los elogios producen mejores resultados en los alumnos flojos o medianamente flojos. Las censuras producen mejores resultados en los alumnos capaces; en los alumnos flojos la censura es inhibitoria y solamente es recomendable cuando sea muy necesaria. Generalmente, las situaciones de censura se deben evitar. El elogio es normalmente superior a la censura.

- Material didáctico: esta debe ser una técnica de motivación para todas las clases donde el maestro se proponga ilustrar y llevar al terreno concreto los asuntos a través de algo más que la palabra (la pizarra, proyecciones cinematográficas, videos, diapositivas...).

- Necesidades del alumno: conocimiento de la utilidad mediata e inmediata de la materia: Las dos son implicativas y constituyen la preocupación constante del maestro. Aunque la utilidad inmediata posea más sentido, también hace falta evidenciar la utilidad mediata de la materia.

- Compañerismo: precisamente el trabajo en grupo nos da un punto clave en la motivación. Como más cohesión tenga el grupo y más sólidas y positivas sean las relaciones afectivas entre sus miembros, con más entusiasmo trabajarán.

- Conocimiento preciso de los objetivos para conseguir: una técnica excelente de motivación es la que da a conocer los objetivos de la unidad y de la lección. Es desanimador el estudio de cualquier cosa sin noción del punto de llegada.

- Reducción de los factores negativos y aumento de los positivos: esta debe ser la norma general de la motivación del aprendizaje. Se deben reducir al mínimo las condiciones desfavorables del trabajo formativo (regaños, críticas exageradas, comparaciones ridiculizantes, exceso de materia de estudio, injusticias y conducta extravagante del maestro) y potenciar al máximo los puntos positivos del alumno (elogios justos, compañerismo, consideración y respeto). 
- Aspiración: una técnica de motivación óptima, aunque difícil de aplicar, es la utilización de las aspiraciones del alumno (nivel de cultura, de vida, de prestigio social...). Esta técnica requiere una indagación de esas aspiraciones, casi siempre inabarcables por el propio alumno, que aún no ha tomado plena conciencia de lo que desea.

- Trabajos graduados: otra manera de motivar consiste en presentar al educando tareas adecuadas y graduadas según las dificultades, partiendo de su nivel de preparación y de capacidad. Mediante esta técnica se conseguirá gran éxito en el propio alumno, cosa que será una motivación excelente.

- Interés por el educando: es una forma de motivación indirecta en la que el maestro se muestra interesado por la personalidad del educando, sus planes de vida, aspiraciones y tareas fuera del ámbito del curso.

- Personalidad del maestro: la personalidad entusiasta, simpática, tolerante y comprensiva del maestro puede actuar indudablemente como factor decisivo de motivación. Si el maestro vive sus clases y hay un clima de interacción afectiva, los alumnos sienten su entrega al trabajo e, indirectamente, a ellos.

\subsubsection{Recomendaciones}

1. El éxito es más incentivador que el fracaso.

2. Los resultados son mejores cuando las tareas se realizan sin coacción.

3. El progreso es más rápido cuando los alumnos reconocen que la tarea coincide con sus intereses más inmediatos.

4. El conocimiento de los resultados es un estímulo fuerte para obtener más rapidez y más exactitud.

5. Los objetivos bien definidos incitan a trabajar.

6. La competición es un recurso de motivación óptimo cuando se la pone en juego en forma grupal o el alumno ejerce con sí mismo (autocompetición).

7. En la motivación, debemos tener en cuenta las diferencias individuales.

8. Solicitado simultáneamente por motivos contradictorios, el educando se muestra perplejo.

9. El papel del maestro no consiste solamente en condicionar motivos nuevos, sino también en explorar convenientemente los abundantes motivos que hay en el educando, ayudándole a relacionarlos con objetivos socialmente valiosos.

10. El maestro debe estar siempre dispuesto a incentivar a los alumnos, ya sea presentando ilustraciones, creando situaciones, planeando trabajos con ellos, comprometiéndoles en actividades individuales o colectivas, escuchándoles y animándoles.

\section{Metodología}

En esta investigación (figura 1), se ha optado por seguir una metodología mixta, cuantitativa y cualitativa, desplegada a lo largo de un trabajo de campo de tres meses. Estas metodologías se han aplicado en coherencia con la naturaleza de la acción educativa a investigar. 


\section{Complemetariedad metodológica \\ Metodologia \\ cuantitativa \\ Diseño casi- \\ experimental \\ Intrumentos (cuantitativa): \\ 1. Pre-test i post-test: prueba de competencias básicas en matemáticas \\ 2. Programa de lectura estartégica de \\ los problemas matemáticos (PLEPM) \\ Metodologia \\ cualitativa \\ Investigación etnográfica \\ Instrumentos (cualitativa) \\ 1. Autoevaluación del alumnado incluida en el PLEPM \\ 2. Entrevista en profundidad profesora}

Figura 1: Metodología de la investigación. Elaboración propia

\section{Desarrollo del programa de Lectura estratégica de los problemas matemáticos} (PLEPM)

El presente artículo aporta una propuesta metodológica sistemática para enfocar la enseñanza y el aprendizaje de la lectura de los problemas matemáticos, que no pretende analizar propiamente el lenguaje matemático, sino la incidencia de la comprensión del lenguaje verbal en la resolución de los problemas matemáticos. Los principios psicopedagógicos (figura 1) que contempla esta propuesta son los siguientes:

a) Prevención: anticipación a una posible dificultad de aprendizaje.

b) Atención a la diversidad: metodología variada para favorecer la equidad en lo referente al acceso al conocimiento.

c) Motivación: Aprendizaje situado, intereses, funcionalidad de los aprendizajes, etc.

d) Fomento del pensamiento crítico ${ }^{1}$ : Enseñar a identificar los problemas contextuales y a darles solución ${ }^{2}$.

e) Globalización y contextualización: hablamos de globalización cuando relacionamos un conocimiento con el mundo y hablamos de globalización cuando nos referimos a su relación con las demás áreas curriculares.

f) Enseñanza estratégica: en esta propuesta de lectura estratégica de los problemas matemáticos se ha optado por la aplicación del modelo de Monereo $^{3}$ (1999),

1 Destacamos a Alsina y Planas (2008); Alcalá (2002) y Vidal (2009) como referentes en problemas que refuerzan el pensamiento crítico.

2 Según Morin (2000), el pensamiento crítico es aquel que estimula la formulación de preguntas pertinentes y la búsqueda de las soluciones adecuadas. Los instrumentos que hay que facilitar para llegar a este pensamiento crítico son muy sencillos de definir, pero difíciles de adquirir: hablar, escuchar, leer y escribir.

3 Los cinco pasos de la secuencia metodológica de Monereo (1999) son: modelaje cognitivo, práctica guiada, práctica en contextos variados, trabajo cooperativo y práctica autónoma. 
añadiendo las condiciones que marcan los principios psicopedagógicos citados. La implementación de la secuencia metodológica implica sistematizar la implementación de ayudas. Cada uno de sus pasos conlleva una carga psicopedagógica acumulativa que podemos asociar al cumplimiento de estos principios. Hay que añadir también que la esencia de esta metodología, de naturaleza metacognitiva, es enseñar y aprender "cómo" tomar consciencia del propio trabajo para lograr realizarlo con éxito.

\section{Relación principios psp / metodología}

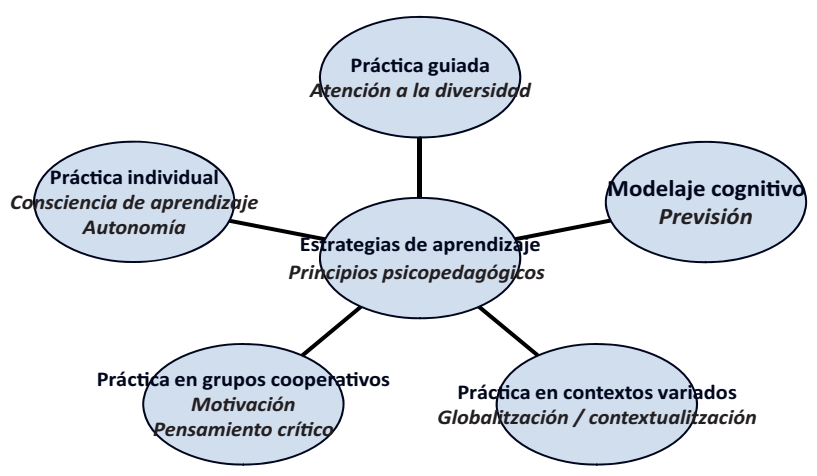

Figura 2: relación entre los principios psicopedagógicos y la metodología estratégica. Elaboración propia.

Como puede deducirse, los cinco pasos de la secuencia metodológica responden a un proceso de enseñanza y aprendizaje graduado en ayudas, que va desde un control total de la lectura por parte del experto (profesor/a), hasta la eliminación total de estas ayudas, cuando el alumno/a consigue la autonomía en la comprensión del texto.

El esquema del Programa de Lectura Estratégica de los Problemas Matemáticos (PLEPM), se puede observar en la figura 3. En él se pone de manifiesto la importancia de la evaluación inicial ${ }^{4}$ para contextualizar y situar al máximo la acción educativa. También se evidencia la existencia de una evaluación final para comprobar si se han logrado los objetivos propuestos.

El modelo presentado se ha experimentado en una investigación ${ }^{5}$ que ha utilizado complementariamente las metodologías cuantitativa, para obtener los datos del rendimiento del alumnado, y cualitativa, para conseguir datos derivados de la autoeva-

${ }^{4}$ La evaluación inicial y final, a nivel de rendimiento cuantitativo, se ha realizado a través de un pre-test i un post-test. Estos datos ofrecen información sobre el nivel de competencias básicas en matemáticas del alumnado de los grupos experimental y control, antes y después de la implementación del programa de lectura estratégica de los problemas matemáticos.

5 Para consultar los datos específicos véase TDX. Balaguer Fàbregas, M.C. (2009) Lectura estratégica dels problemes matemàtics a l'Educació Primària. Tesi doctoral en xarxa, Universitat Internacional de Catalunya. 
luación cualitativa del alumnado y datos relacionados con las reflexiones de la profesora que ha implementado el programa.

\section{MODELO DE LECTURA ESTRATÉGICA DE LOS PROBLEMAS MATEMÁTICOS}

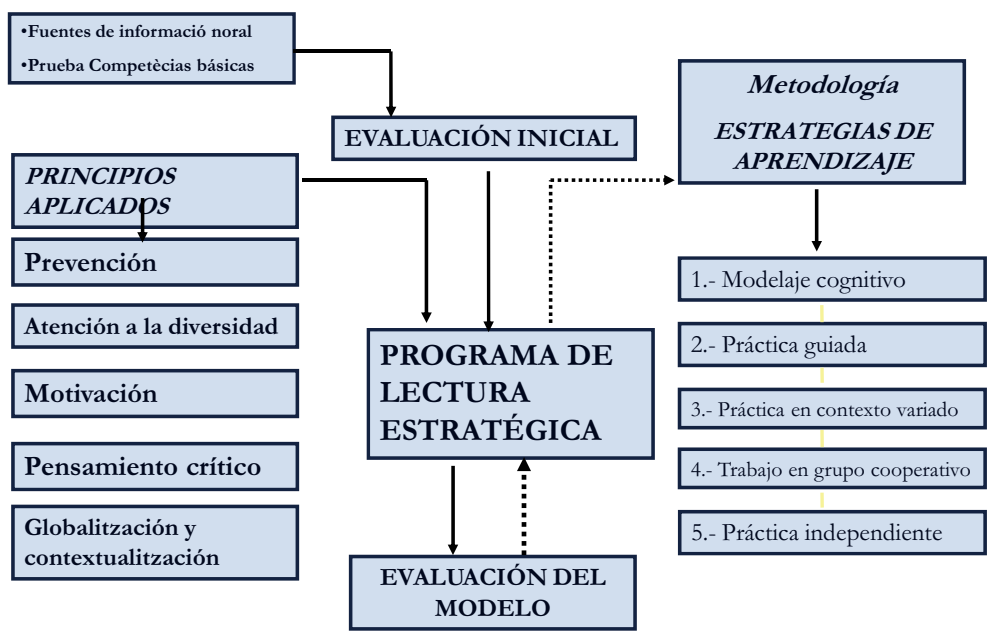

Figura 3: esquema del Programa de Lectura Estratégica de los Problemas Matemáticos (PLEPM). Elaboración propia.

\section{Conclusiones}

Las conclusiones que se desprenden de esta investigación son las siguientes:

La mejora de la comprensión lectora no pasa, en absoluto, por un refuerzo de su automatización, al menos en la etapa de Educación o primer ciclo de Educación Primaria. Más bien al contrario. Es preciso desarrollar un conjunto de medidas que permitan el fomento de actitudes positivas y comportamientos activos que respeten la naturaleza infantil. Por ejemplo, el uso del lápiz para subrayar o hacer anotaciones que den soporte a la autoregulación de la comprensión.

Es importante enseñar hábitos de naturaleza metacognitiva para trabajar la comprensión desde la Educación Infantil. Algunos de estos hábitos son: la relectura, hacer hipótesis, autocuestionarse la comprensión, verificar las hipótesis, hacer inferencias, relacionar con los conocimientos previos, leer hacia adelante y hacia atrás, acostumbrarse a parafrasear, aunque sean textos muy pequeños, etc. Es interesante habituar a los niños/as al mantenimiento de una o más ideas en la memoria de trabajo. Los hábitos mencionados están íntimamente relacionados con los hábitos del esfuerzo metal: "pensar", "relaciona", "comparar", "argumentar", etc.

La intervención educativa para mejorar la comprensión lectora precisa la aplicación de estrategias verticales y también longitudinales. Las verticales son necesarias para estructurar las sesiones en base a un "antes", "durante" y "después". Cada paso servirá para trabajar respectivamente, la activación de los conocimientos previos e 
identificación de elementos gráficos que aporten información, el desarrollo del nuevo aprendizaje y la autoevaluación.

Las estrategias longitudinales se precisan para la aplicación de una secuencia metodológica flexible que sistematice la administración y la retirada gradual de ayudas en función de la autonomía en la lectura que el alumnado vaya adquiriendo.

Las ayudas impartidas por parte del profesorado o de otros/as alumnos/as han de ser de naturaleza metacognitiva. Su objetivo tiene que ser favorecer el desarrollo de estrategias de autoregulación de la lectura, llegando a ser cada alumno/a responsable de su comprensión. Es sustancial enseñar y pautar las estrategias que utilizan los expertos para comprender, a los aprendices. A menudo, hay niños o niñas que son incapaces de deducirlas en solitario.

La lectura comprensiva de los problemas matemáticos no tiene porqué ser distinta de la que se realiza en las demás áreas. Al contrario, hay que fomentar un tratamiento único y transversal. Un elemento idiosincrático del área matemática puede ser la enseñanza y aprendizaje de las estrategias de organización de la información.

Por último, en la comprensión de la lectura de los problemas matemáticos se ha manifestado como muy adecuado, favorecer la relectura espontanea y la discusión en grupo, acciones que aseguran la comprensión como base para solucionar esos problemas.

\section{Referencias bibliográficas}

ALCALÁ, Manuel (2002): La construcción del lenguaje matemático. Barcelona, Graó

ALSINA, Ángel y PLANAS, Nuria (2008): Matemática inclusiva. Propuestas para una educación matemática. Madrid, Narcea.

AUSUBEL, David. P. (2002): Adquisición y retención del conocimiento: una perspectiva cognitiva. Barcelona, Paidós Ibérica.

BALAGUER, Carme (2009): Lectura estratègica dels problemes matemàtics a l'Educació Primària. Tesis doctoral en xarxa (TDX), Universitat Internacional de Catalunya.

BREWER, William F. y NAKAMURA, Glenn V. (1984): "The nature and function of schemas". En R.S. Wyer y T.K. Skrull (eds.) Handbook os social cognition. Hillsdale, NJ, Erlbaum.

CORBALÁN, Fernando (1995): La matemática aplicada a la vida cotidiana. Barcelona, Graó.

GAIRÍN, Joaquín (1990): Las actitudes en educación. Barcelona, Boixareu Universitaria.

GARDNER, Martin (1993): Miscelania matemática. Barcelona, Salvat (Biblioteca científica).

MAGER, Robert (1981): Actitudes positivas en la enseñanza. Pax, México

MONEREO, Carles (coord., 1999): Estrategias de enseñanza y aprendizaje. Formación del profesorado y aplicación en la escuela. Barcelona, Graó 
MONEREO, Carles y CASTELLÓ, Montserrat (1997): Las estrategias de aprendizaje. Cómo incorporarlas a la práctica educativa. Barcelona, Edebé.

MORIN, Edgar (2000): La mente bien ordenada. Barcelona, Seix Barral.

PIAGET, Jean (1985): El nacimiento de la inteligencia en el niño. Barcelona, Crítica

ROGOFF, Barbara (1993): Aprendices del pensamiento. El desarrollo cognitivo en el contexto social. Barcelona, Paidós

RUMELHART, David E. (1980): "Schemata: the building block of cognition". En R.J. Spiro, B.C. Bruce y F. Brewer (eds.). Theoretical issues in Reading comprehension. Hilldale, NJ: Erlbaum

VIDAL, Salvador (2009): Estrategias para la enseñanza de las matemáticas en secundaria. Barcelona, Laertes.

VIDAL, Salvador (2010): “Talens dag att skapa lust för matematiklärande”, en Nämnaren Tidskrift för matematikundervisning (Göteborgs universitet) 173 (1), pp. 4346.

VIDAL, Salvador (2010): "La comunicación en la didáctica de las matemáticas”, en Vivat Academia. (UCM). 112, pp.1-9

VIDAL, Salvador (2011): “Good morning, numbers day", en Australian Primary Mathematics Classroom. (The Australian Association of Mathematics Teachers Inc). 16 (3), pp. 25-28.

VIGOTSKY, Lev S. (1979): El desarrollo de los procesos psicológicos superiores. Barcelona, Crítica

\section{Salvador VIDAL RAMÉNTOL}

Universidad Internacional de Catalunya

Profesor Agregado de Didáctica de las Matemáticas en el Grado de EP de la Facultad de Educación. Profesor del Máster de Investigación. Miembro de la comisión de doctorado de la UIC. Universitat Internacional de Catalunya. Dr. en Ciencias de la Educación por la UB svidal@uic.es

\section{María Carmen BALAGUER FÀBREGAS}

Universidad Internacional de Catalunya

Facultat d'Educació

Profesora adjunta de Aprendizaje Matemático en la EI. Doctora en Humanidades por la UIC

mcbalaguer@uic.es 